\title{
Arbeitsbelastung durch Anästhesiegase und chirurgische Rauchgase und Schutzmaßnahmen im chirurgischen Operations(OP-)bereich - was der Chirurg wissen sollte
}

\author{
Work Strain by Anaesthetic Gas and Surgical Smoke due to Tissue Coagulation as well as \\ Safety Measures in Surgical Operating Rooms - What the Surgeon Needs to Know
}

Autoren

Institute
I. Boeckelmann ${ }^{1}$, S. Sammito ${ }^{1}$, F. Meyer ${ }^{2}$

${ }^{1}$ Bereich Arbeitsmedizin, Otto-von-Guericke-Universität, Medizinische Fakultät, Magdeburg, Deutschland

${ }^{2}$ Klinik für Allgemein-, Viszeral- \& Gefäßchirurgie, Universitätsklinikum Magdeburg A.ö. R., Magdeburg, Deutschland

\section{Schlüsselwörter \\ - OP-Personal \\ - Arbeitsbelastung \\ - Anästhesiegase \\ - chirurgische Rauchgase \\ Key words \\ operating room personnel \\ - work strain \\ - anaesthetic gases \\ - surgical smoke gases}

Bibliografie

DOI http://dx.doi.org/ 10.1055/s-0032-1328179 Zentralbl Chir 2013; 138: 94-103 @ Georg Thieme Verlag KG Stuttgart · New York . ISSN 0044-409X

Korrespondenzadresse Prof. Dr. Irina Boeckelmann Otto-von-Guericke-Universität Medizinische Fakultät Bereich Arbeitsmedizin Leipziger Straße 44 39120 Magdeburg Deutschland Tel.: $0391 / 6715059$ Fax: 0391/6715083 irina.boeckelmann@ med.ovgu.de

\section{Zusammenfassung}

$\nabla$

Die Exposition der Atemwege während chirurgischer Eingriffe ist ein relevantes aber wenig erforschtes Thema der Arbeitsmedizin. Auf der Basis einer Literaturrecherche werden die relevanten Informationen zu den möglichen gesundheitlichen Risiken zusammengefasst. Im Operationssaal kann es zu einer Exposition der Atemwege durch Gase (volatile Anästhetika) und Aerosole (Rauchgase durch Koagulation) kommen. Diese Exposition stellt ein potenzielles Gesundheitsrisiko dar, wenn die Schutzmaßnahmen ungenügend sind. $\mathrm{Zu}$ den möglichen Gesundheitsstörungen gehören Schwangerschaftsrisiken, Fertilitätsstörungen, Neurotoxizität und Krebsentwicklung. Diese Gesundheitsstörungen werden durch Primärprävention vorgebeugt und frühzeitig durch arbeitsmedizinische Vorsorgeuntersuchungen (Sekundärprävention) erkannt. Entsprechende Schutzmaßnahmen werden nach dem STOP-Prinzip (Substitution, Technische, Organisatorische und Persönliche Maßnahmen) entwickelt. Die Gefährdungsbeurteilung beginnt mit der Beschreibung der Arbeitsverfahren und der Ermittlung der toxischen Eigenschaften der verwendeten Arzneimittel und Medizinprodukte. Mittels dieser Daten und/oder durch Bestimmung der individuellen Exposition kann dann das Gefährdungspotenzial bewertet werden. Daraus werden die notwendigen Arbeitsschutzmaßnahmen abgeleitet und die Arbeitsorganisation optimiert. Das Operationspersonal wird in die angezeigten präventiven Verhaltensweisen eingewiesen. Infolge der besseren Umsetzung der obigen Prinzipien, durch die Einführung neuer Narkosemittel und technologische Entwicklungen sind die potenziellen Belastungen über die Atemwege im Operationssaal in den letzten 10 Jahren reduziert worden. So werden Schwangerschaftsrisiken und Fertilitätsstörungen durch die Exposition volatiler Narkosemitteln jetzt als gering eingeschätzt. Die

\section{Abstract}

Exposure of the respiratory tract during surgical interventions is an important topic of occupational medicine, which has only rarely been investigated. Based on a literature search, relevant information on the possible health risk is summarised. Within the operating room, an exposure of the respiratory tract to gas (volatile anaesthetics) and aerosols (smoking gas by coagulation) can occur. This exposure needs to be considered as a potential health risk if safety measures are not sufficient. Health risks comprise possible disturbances of gravidity and fertility, neurotoxicity and cancer generation. Such health risks can be prevented with primary preventive measures and can be early recognised/diagnosed by preventive investigations of occupational medicine (secondary prevention). Safety measures are developed according to the STOP principle (substitution, technical, organisatory and personal measures). Assessment of the potential danger begins with an appropriate description of the working procedure and detection of the toxic features of the drugs and medical products, which helps to determine individual exposure and to estimate risk potential. Required occupational safety measures can be derived from this and, subsequently, the work organisation can be optimised. In addition, employees in the operating room are to be instructed about the indicated preventive mode of behaviour. Due to better implementation of the abovementioned basic principles, introduction of novel narcotics and technological developments, potential exposure of the respiratory tract within the operating room has been reduced over the last 10 years. Thus, risks for gravidity and possible disturbances of fertility by exposure to volatile narcotics are currently assessed to be low. However, available data on health risks of the chronic exposure to smoking gases are still deficient although toxic and cancerogenic organic pyrolysis products 
Datenlage zu den gesundheitlichen Risiken der chronischen Exposition zu Rauchgasen bleibt aber ungenügend, obwohl toxische und krebserregende organische Pyrolyseprodukte erzeugt werden. Der Schutzeffekt moderner Lüftungssysteme (wie z.B. Laminar-Airflow) ist wenig untersucht worden. Zu diesen Fragen sind weiterführende Studien notwendig. are generated. The protection effect of modern air conditioning (e.g., laminar air flow) is only insufficiently investigated. Therefore, further studies on these problems are required.

\section{Einleitung}

\section{$\nabla$}

Die betriebsmedizinische Betreuung der chirurgisch tätigen Beschäftigten im Gesundheitsdienst ist nicht nur auf Infektionsprophylaxe, Hautschutz, Prävention der Wirbelsäulenerkrankungen, Früherkennung psychischer Belastung und Ergonomie der Gerätetechnik im Operations(OP-)saal begrenzt [1-3]. Ein weiteres Feld der arbeitsmedizinischen Vorsorge und Forschung ist die Problematik der Narkose- und Rauchgaseexposition während chirurgischer Eingriffe [4-5].

In Krankenhäusern wird eine große Anzahl von chemischen Stoffen verwendet, die nach dem Chemikaliengesetz (ChemG) zur Reihe „Gefahrstoffe“ einzuordnen sind und die negative Effekte auf die Gesundheit ausüben können. Hierzu gehören Narkoseund Rauchgase bzw. diverse Aerosole, die eine bisher nicht gebührend berücksichtigte Rolle spielen.

In der Literatur wird immer wieder kontrovers diskutiert, ob die chronisch subanästhetischen Konzentrationen der Narkosegase wirklich schädigende Wirkungen hervorrufen können [6-10]. Die Belastung des OP-Personals durch chronische Exposition von Anästhesiegasen am Arbeitsplatz wird dabei wohl unterschätzt [8]. Insbesondere liegen kaum Studien zu den Inhalationsanästhetika Desfluran und Sevofluran vor, die in höheren Konzentrationen eingesetzt werden und so eine höhere Belastung des OPPersonals zu erwarten wäre.

Eine neuartige, weitere Belastung im OP-Bereich stellen verschiedene chirurgische Verfahren dar, die Rauchgase erzeugen. Nur einige Verfahren sind hier stellvertretend an dieser Stelle zu nennen: Elektrokauter, Laser, Ultraschallskalpell u. a. [11]. Auch diese Gase bzw. deren Bestandteile können potenziell aufgrund der toxischen Eigenschaften gesundheitsschädliche Auswirkungen auf das medizinische Personal haben. Konkrete Auswirkungen auf das OP-Personal sind auch hier bisher wenig untersucht und belegt [12].

Das Ziel dieser kompakten Kurzübersicht besteht darin, basierend auf einer selektiven Literaturrecherche und arbeitsmedizinisch zusammengefassten prinzipiellen Aspekten zum Thema

- die chirurgisch tätigen Kollegen (natürlich neben dem übrigen OP-Personal) zur Problematik der Belastung und Gefährdung durch Anästhesiegase bzw. chirurgische Rauchgase zu sensibilisieren (obwohl die meisten raumlufttechnischen Anlagen [RLT] nach nationalen Vorgaben [DIN 1946 Teil 4] in medizinischen Behandlungsräumen ausreichend sind) sowie

- ein Basiswissen zu dieser Problematik zu konsolidieren. Des Weiteren werden die Schutzmaßnahmen nach dem in der Arbeitsmedizin etablierten STOP-Prinzip (Substitution, Technische, Organisatorische und Persönliche Maßnahmen) für die beiden Belastungsarten aufgeführt.

Es werden in dieser Arbeit auch heute nicht mehr verwendete Anästhetika besprochen und die Problematik der chronischen Exposition beim OP- und Anästhesiepersonal erläutert, da 1. die älteren Kollegen bei Ausübung ihrer Tätigkeit damals potenziell dieser chronischen Belastung ausgesetzt waren,
2. sich der Leserkreis nicht nur auf die in Deutschland tätigen Mediziner beschränkt und

3. in anderen Ländern Anästhetika wie Halothan immer noch verwendet werden.

\section{Allgemeine Vorbetrachtungen \\ $\nabla$}

Eine der wichtigsten Aufgaben der Arbeitsmedizin ist es, Arbeitsunfälle und Berufskrankheiten der Arbeitnehmer zu verhindern, d.h. arbeitsbedingten Gesundheitsstörungen rechtzeitig unter Anderem durch Primärprävention vorzubeugen und diese frühzeitig durch arbeitsmedizinische Vorsorgeuntersuchungen (Sekundärprävention) zu erkennen.

Die verschiedenen rechtlichen Vorschriften und Gesetze in Deutschland wie z.B. das Arbeitsschutzgesetz (ArbSchG) verlangen von den Arbeitgebern, dass alle notwendigen Arbeitsschutzmaßnahmen getroffen werden müssen, um die Arbeitnehmer und deren Gesundheit vor beeinträchtigenden und gefährdenden Einwirkungen bei der Arbeit zu schützen. Das betrifft auch den Arbeitsplatz „(chirurgischer) OP-Bereich“. Die Arbeitnehmer, also das chirurgische OP- und Anästhesiepersonal, sollen den Arbeitgeber dabei unterstützen. Auch die Arbeitnehmer haben nach ArbSchG Rechte und Pflichten, z.B. die Pflicht, alle festgestellten Mängel am Arbeitsplatz, die Auswirkungen auf Sicherheit und Gesundheit haben können, dem Arbeitgeber zu melden. Wenn der Arbeitgeber nicht reagiert, ist eine Meldung an die zuständige Behörde ratsam. Dem Arbeitnehmer dürfen nach dem ArbSchG hierdurch keine Nachteile entstehen.

Ein Betriebsarzt im Krankenhaus sollte in enger Zusammenarbeit mit der Fachkraft für Arbeitssicherheit eine Gefährdungsbeurteilung an den Arbeitsplätzen aller Narkosebereiche durchführen ( $§ 5$ „Beurteilung der Arbeitsbedingungen“ und $\S 6$ „Dokumentation“ ArbSchG). Hierdurch soll die Gefährdung unter Berücksichtigung der jeweils arbeitsplatzspezifischen Bedingungen abgeschätzt und erforderliche Maßnahmen des Arbeitsschutzes ermittelt werden ( $\triangle$ Abb. 1 und 2 ).

Eine der ersten arbeitshygienischen Messungen im OP-Bereich war die von dem Dresdener Chirurgen Kelling im Jahr 1918 im Zentralblatt für Chirurgie beschriebene Kontrollmessung der Narkosedämpfe im OP-Saal [13]. Zwei weitere Wissenschaftler, Arbeitshygieniker aus Berlin, Hirsch und Kappurs, beschäftigten sich ebenfalls mit dieser Thematik und zeigten fast 10 Jahre danach, dass bei der Luftkonzentration in OP-Sälen durch Narkoseäther chronische Schäden nicht auszuschließen sind [14].

Auch in der heutigen Zeit ist dieses Thema für Arbeitsmediziner von hohem Interesse, da sowohl der Inhalations- als auch der Kombinationsnarkose (Anästhesiegase in Verbindung mit intravenösen [i.v.] Analgetikamitteln) das folgende gemeinsame Problem zugrunde liegt: Die anästhesiegasführenden Geräte weisen oft eine Leckage auf [10]. Das bedeutet, dass das OP-Personal kontinuierlich dieser Belastung ausgesetzt wird.

Die klassische Gefährdungsbeurteilung am Arbeitsplatz OPRaum bei Exposition durch Narkosegase und/oder chirurgische 


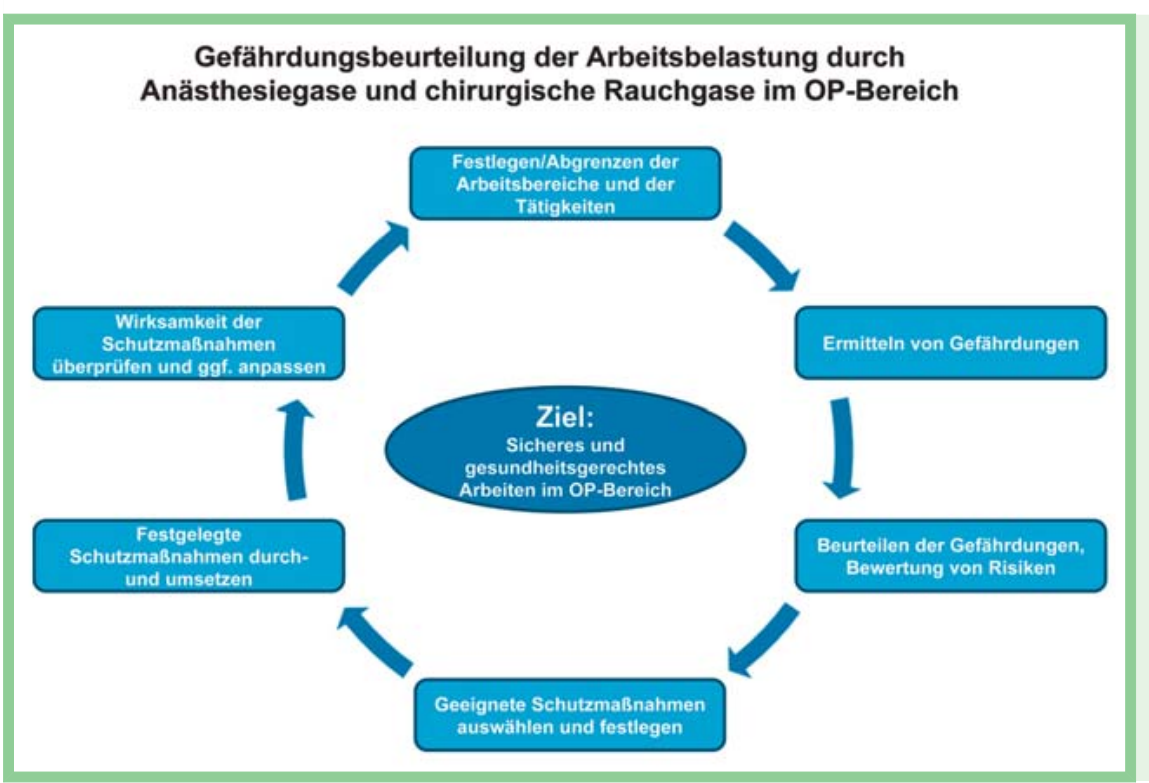

Abb. 1 Gefährdungsbeurteilung der Arbeitsbelastung durch Anästhesiegase und chirurgische Rauchgase im OP-Bereich.

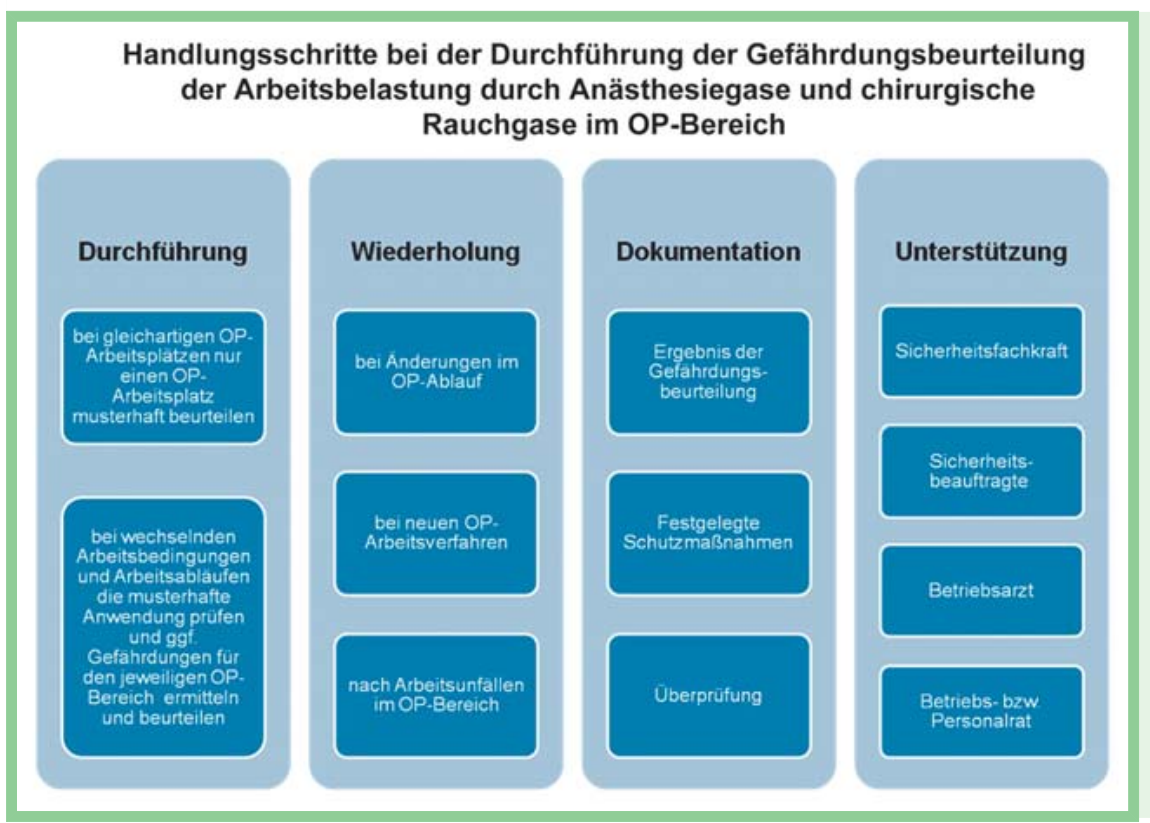

Abb. 2 Handlungsschritte bei der Durchführung der Gefährdungsbeurteilung der Arbeitsbelastung durch Anästhesiegase und chirurgische Rauchgase im OP-Bereich.

Rauchgase beginnt mit der Ermittlung der toxischen Eigenschaften der am OP-Arbeitsplatz verwendeten Medizinprodukte und der Beschreibung der Arbeitsverfahren sowie Arbeitsorganisation. Mittels dieser Daten und/oder Bestimmung der individuellen Exposition eines Beschäftigten kann man die vorliegende Exposition bewerten und notwendige Arbeitsschutzmaßnahmen festlegen als auch die Arbeitsorganisation optimieren. In der Arbeitsmedizin hat sich das STOP-Prinzip als Kriterium der Wahl von Schutzmaßnahmen in der Reihenfolge etabliert ( $\bullet$ Abb. 3):

S - Substitution von Gefahrstoffen,

$\mathbf{T}$ - Technische Maßnahmen,

$\mathbf{O}$ - Organisatorische Maßnahmen,

P - Persönliche Maßnahmen.

Bei der Gefährdungsbeurteilung des potenziell exponierten OPPersonals und des Arbeitsplatzes soll der Betriebsmediziner neben den toxischen Eigenschaften einer Substanz auch die Anzahl von zahlreichen Einflussfaktoren berücksichtigen, die die Toxizi- tät volatiler Anästhetika und chirurgischer Rauchgase verändern können. Dazu gehören:

- OP-Raum-Größe,

- Art der Raumbelüftung,

- Luftwechselrate,

- Art der Patienten,

- Art der OP,

- Typ des verwendeten Narkosesystems (geschlossenes, halb offenes oder halb geschlossenes),

- Frequentierung der durchgeführten OPs pro Tag bzw. Woche und

- Routine des Anästhesie- und des OP-Personals [1]. 


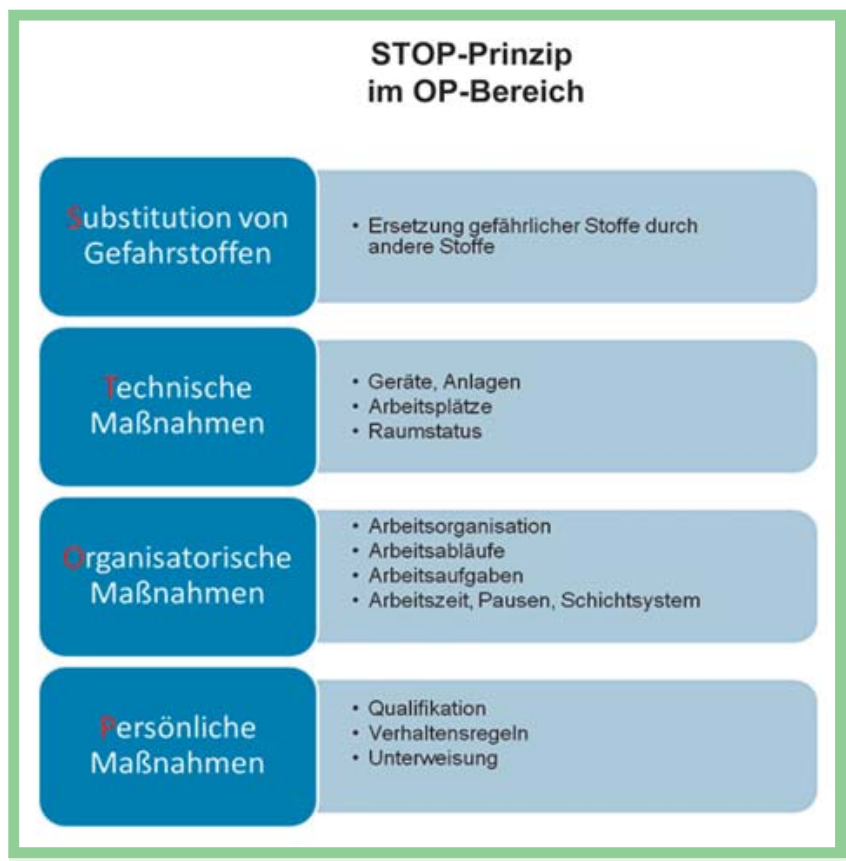

Abb. 3 STOP-Prinzip im OP-Bereich.

\section{Anästhesiegase \\ $\nabla$}

Im Allgemeinen werden in der Chirurgie bei operativen Eingriffen Allgemeinnarkosen oder Regionalanästhesieverfahren verwendet. Zu den wichtigsten in den letzten 30 Jahren verwendeten Narkosegasen im OP-Bereich mit einer potenziellen Gesundheitsgefährdung gehör(t)en halogenierte Kohlenwasserstoffe (Halothan: 2-Brom-2-Chlor-1,1,1-Trifluorethan oder Fluothane) und Narkosegase aus der Ethergruppe (Enfluran) bzw. gehören das heutzutage immer noch eingesetzte Distickstoffmonoxid $\left(\mathrm{N}_{2} \mathrm{O}\right)$ und Narkosegase aus der Ethergruppe (Desfluran, Sevofluran und Isofluran). Die beiden gasförmigen Anästhetika Desfluran und Sevofluran zeichnen sich durch niedrige Blut-Gas-Verteilungskoeffizienten und sehr gute Verträglichkeit aus. Sie gelten deshalb heute als Standardanästhetika. Halothan hingegen wird heute in Deutschland kaum mehr verwendet, findet jedoch in der Kinderanästhesie doch noch eine breite Anwendung. Die Vorteile der reinen Inhalationsanästhesie liegen in der guten Steuerbarkeit und in der Elimination der Substanzen unabhängig von Leber- und Nierenfunktion [15]. Als Nachteil ist allerdings eine potenzielle Organtoxizität zu nennen [16].

\section{Anästhesiegase und deren Wirkung}

Es existiert eine Reihe von Publikationen, die sich mit dem Thema Belastungen durch Anästhesiegase bei OP-(und/oder Anästhesie-)Personal befassen [6, 8,17-29].

Die Wirksamkeit der Inhalationsanästhetika bei Patienten ist gut erforscht; unerwünschte Begleiterscheinungen und Nebenwirkungen der Anästhesiegase, u.a. die Halothanhepatitis, wurden beschrieben [9]. Auch für das exponierte OP- und Anästhesiepersonal wurde über die möglichen Gesundheitsbeeinträchtigungen und -risiken berichtet. Das OP-Personal ist zwar deutlich niedrigeren Konzentrationen von Anästhesiegasen ausgesetzt, jedoch dafür während eines längeren Zeitraums. Die Belastung summiert sich über den Arbeitstag ( $8 \mathrm{~h}$ und mehr) und mehrere Arbeitsjahre. Daher muss die Gefährdungsbeurteilung nach Ände- rung des arbeitsbedingten Vorgehens (Verfahren, Technik) immer wieder neu und aktualisiert durchgeführt werden.

Bei der Anwendung des Anästhesiegases Distickstoffmonoxid (syn.: $\mathrm{N}_{2} \mathrm{O}$, Lachgas, Distickstoffoxid, Stickoxydul) sind folgende Effekte beim OP- und/oder Anästhesiepersonal durch berufliche Expositionen in der Literatur beschrieben worden:

- periphere Neuropathien [30],

- vermehrte neurologische Beschwerden (bei Zahnärzten und Zahnarztgehilfinnen, die einer chronischen Lachgasexposition während mehr als $6 \mathrm{~h}$ pro Woche ausgesetzt waren und an deren Arbeitsplatz eine Gasabsaugungsanlage fehlte) [31],

- Beschwerdezunahme wie Kopfschmerzen, Müdigkeit und Schwindel, wobei die Beschwerdehäufigkeit des OP-Personals mit der Leistung der Raumventilation im OP-Saal korrelierte [32],

- Abnahme der Reaktionszeit beim OP-Personal $[32,33]$, wobei das in anderen Studien nicht bestätigt werden konnte [34-36],

- Beeinträchtigung mentaler Funktionen bei halbstündigen Expositionen bei Lachgaskonzentrationen von 20\% [37],

- Veränderungen im Immunsystem wie z. B. verminderte Anzahl von B-Lymphozyten und natürlichen Killerzellen beim Anästhesiepersonal [38], wobei in einer anderen Studie [39] demgegenüber keine Immunsuppression in der Art der untersuchten Klientel nachgewiesen wurde,

- erhöhte Häufigkeit von Nieren- und Leberschädigungen bei exponiertem Personal [40],

- Zusammenhang zwischen Exposition beim OP-Personal und Hepatitis [41] sowie

- verminderte Fertilität durch Lachgasexposition [42] - über die Spontanabortraten wird kontrovers diskutiert [9].

Die Untersuchungen von Zühlsdorff [29] zeigten, dass die Konzentrationen von Lachgas die vorgeschriebenen Höchstwerte von 100 ppm während einer OP deutlich überschritten. Lachgas wird auch heute noch zur Supplementierung von volatilen und i.v. Anästhesien eingesetzt [29].

Man findet in der Literatur einige Hinweise dafür, dass infolge beruflicher Belastung durch Halothan solche Beeinträchtigungen des Befindens wie Müdigkeit, Nervosität, Kopfschmerzen, Konzentrations- und Antriebsstörungen und die Verschlechterung des Leistungsvermögens vorkommen können $[37,43]$. Bei Krankenhauspersonal, das gegenüber volatilen Gasen chronisch exponiert war, wurden Gesundheitsstörungen wie Leberparenchymschäden, Irritationen der Atemwege, gastrointestinale Beschwerden, Herzrhythmusstörungen u.a. beobachtet $[19,44,45]$. Es wurde in den Studien auch über Befindensstörungen wie Ermüdbarkeit, Nervosität, Irritierbarkeit, Kopfschmerzen und Benommenheit berichtet. In einer Fallbeschreibung wurde ein schweres Asthma bei einem Anästhesisten dargestellt [46]. Toxischer Einfluss auf die DNA-Synthese wird durch Conzen [7] berichtet. Einige epidemiologische Studien konnten zeigen, dass beim Anästhesiepersonal eine erhöhte Inzidenz an Leukämien, Lymphomen und anderen Malignomen aufgetreten ist. Dem steht eine Reihe anderer Studien gegenüber, in denen kein erhöhtes kanzerogenes Risiko beim OP-Personal gefunden wurde [9]. Nur wenige dieser Studien beschreiben ausführlich die Untersuchungsbedingungen und Klimatisierungsparameter wie z.B.:

- die Fläche des OP-Saales,

- das Gesamtvolumen des Raumes,

- die Frischluftzufuhr für diesen Raum,

- Luftwechsel pro Stunde ohne Rückführung der Umluft,

- die Beschreibung der Zuluftströmung oder

- den Ort der Luftabsaugung. 
Tab. 1 Aktuelle Grenzwerte für Anästhesiegase (Grenzwertliste 2011 IFA-Report 1/2011 der DGUV [47]).

\begin{tabular}{|c|c|c|c|c|c|}
\hline \multirow[t]{2}{*}{ Anästhetika } & \multicolumn{2}{|c|}{ Arbeitsplatzgrenzwert (AGW) ${ }^{1}$} & \multirow[t]{2}{*}{ Spitzenbegrenzung ${ }^{2}$} & \multirow[t]{2}{*}{ Einstufung/Bewertung ${ }^{3}$} & \multirow[t]{2}{*}{ Biologischer Grenzwert (BGW) ${ }^{4}$} \\
\hline & {$\left[\mathrm{ml} / \mathrm{m}^{3}\right]$} & {$\left[\mathrm{mg} / \mathrm{m}^{3}\right]$} & & & \\
\hline $\begin{array}{l}\text { Lachgas (Distick- } \\
\text { stoffmonoxid) }\end{array}$ & 100 & 180 & $2(I I)$ & $Y$ & - \\
\hline Halothan & 5 & 41 & $8(I I)$ & $R_{E} 2$ & + (Trifluoressigsäure 2,5 mg/l im Blut) \\
\hline Enfluran & 20 & 150 & $8(I I)$ & $\mathrm{Y}$ & - \\
\hline Isofluran & 10 & 77 & - & - & - \\
\hline Desfluran & - & - & - & - & - \\
\hline Sevofluran & - & - & - & - & - \\
\hline
\end{tabular}

1 Arbeitsplatzgrenzwert (AGW) bezieht sich auf die Konzentration (Gewichts- bzw. Volumenanteil) eines Gefahrstoffs in der Luft am Arbeitsplatz. AGW-Werte sind Schichtmittelwerte bei in der Regel täglich 8-stündiger Exposition und bei Einhaltung einer durchschnittlichen Wochenarbeitszeit von $40 \mathrm{~h}$.

2 Spitzenwert oder Überschreitungswert: Diese Kurzzeitwerte ergänzen die AGW, indem sie die Konzentrationsschwankungen um den Schichtmittelwert nach oben hin sowie in ihrer Dauer und Häufigkeit beschränken. Die Kurzzeitwertkonzentration ergibt sich aus dem Produkt von AGW und Überschreitungsfaktor; z. B. bei 8-facher Überschreitung des AGW 4-mal pro Schicht über 15 Minuten darf in einer Schicht keine weitere Exposition mehr erfolgen, da sonst das Produkt aus Schichtlänge und AGW überschritten wird. Für die Intervalle zwischen den Perioden mit einer Konzentration oberhalb des AGW (Kurzzeitwertphase) ist ein Zeitraum von 1 Stunde anzustreben. Insgesamt sind 4 Kurzzeitwertphasen innerhalb einer Schicht zulässig. Kategorie II = resorptiv wirksame Stoffe. Als Basiswert (15-Minuten-Mittelwert) wird ein Überschreitungsfaktor von 2 festgelegt, der stoffspezifisch angepasst werden kann (bis max. 8). Die betriebliche Überwachung soll durch messtechnische Mittelwertbildung über 15 Minuten erfolgen, z. B. durch eine 15-minütige Probennahme. Bei Stoffen der Kurzzeitwert-Kategorie II sind auch längere Überschreitungsdauern zulässig, solange das Produkt aus Überschreitungsfaktor (ÜF) und Überschreitungsdauer eingehalten wird (Beispiel: Bei einem ÜF von 8 ist auch ein ÜF 4 über 30 min oder ein ÜF 2 über 60 min möglich).

3 Einstufung/Bewertung nach der GHS (CLP)-Verordnung 127 2/2008, Anhang VL bzw. der Technischen Regel für Gefahrstoffe (TRGS 905); Risikogruppe für Schwangere (TRGS 905; Anhang I GefStoffV): RE 2-Stoffe - Stoffe, die als fruchtschädigend (entwicklungsschädigend) für den Menschen angesehen werden sollten, Y-Stoffe - Stoffe, bei denen ein Risiko der Fruchtschädigung bei Einhaltung des Arbeitsplatzgrenzwerts (AGW) und des biologischen Grenzwerts (BGW) nicht befürchtet zu werden braucht.

${ }^{4}$ Biologischer Grenzwert (BGW) - die bei Menschen höchstzulässige Quantität eines Gefahrstoffs bzw. eines Gefahrstoffmetaboliten oder eines Beanspruchungsindikators, die nach dem gegenwärtigen Stand der wissenschaftlichen Kenntnis im Allgemeinen die Gesundheit der Beschäftigten nicht beeinträchtigt. Sie bezieht sich wie AGW auf eine Arbeitszeit von 8 Stunden täglich und 40 Stunden wöchentlich. Probennahmezeitpunkt: Expositionsende bzw. Schichtende; bei Langzeitexposition: nach mehreren vorangegangenen Schichten.

Die Literaturrecherche zeigt, dass die Exposition durch volatile Anästhetika für das OP-Personal ein potenzielles Risiko darstellen kann. Es ist bei ungünstigen arbeitshygienischen Bedingungen am OP-Arbeitsplatz mit Befindlichkeitsstörungen und leichten neuropsychologischen Beeinträchtigungen zu rechnen [9]. Die nationalen Institutionen verschiedener Länder (die schweizerische Unfallversicherungsanstalt SUVA, das französische Institut National de Recherche et de Securité IRNS und die deutsche Berufsgenossenschaft für Gesundheitsdienst und Wohlfahrtspflege BGW) kamen jedoch zum Konsens, dass „Schwangerschaftsrisiken und Fertilitätsstörungen eher geringer einzuschätzen sind“ [26].

In dem Merkblatt zur Berufskrankheit Nr. 1302 „Erkrankungen durch Halogenkohlenwasserstoffe“ sind die Gefahrenquellen aufgelistet. Zu diesen chemischen Verbindungen mit einer gesundheitlichen Gefährdung gehören Narkosemittel wie Enfluran und Halothan.

Die statistischen Daten zeigen aber, dass die Berufskrankheiten wie Nephropathien durch Methoxyfluran, Asthma bronchiale durch Enfluran oder Hepatitiden durch Halothan beim Personal im Gesundheitswesen sehr selten vorkommen [9].

\section{Arbeitshygienische Beurteilung der Exposition durch volatile Anästhetika}

Westphal et al. [8] kritisieren, dass zur Reduzierung der Schadstoffbelastung das Ausweichen auf „neue“ Inhalationsanästhetika empfohlen wird. Für diese volatilen Anästhetika gibt es aber keine vom Gesetzgeber festgelegten Grenzwerte, da deren toxische Potenzen noch nicht ausreichend erforscht wurden und feststehen.

Der Arbeitsmediziner und die Sicherheitsfachkraft sollten daher Wege suchen, um dieses potenzielle Risiko suffizient zu beurteilen und ggf. zu senken, z.B. durch die Reduzierung der Belastung durch Anästhesiegase beim exponierten OP-Personal. Die arbeitshygienischen Grenzwerte zumindest für die Gase mit be- kannten Grenzwerten ( $\bullet$ Tab. 1) sollten dazu peinlichst eingehalten werden. Sie bilden auch eine wichtige Grundlage zur Gefährdungsbeurteilung am Arbeitsplatz.

Arbeitsplatzgrenzwerte sind an die frühere maximale Arbeitsplatzkonzentration (MAK) angelehnt, die biologischen Grenzwerte auf die früheren BAT-Werte (Biologischer Arbeitsstoff-Toleranzwert) bezogen [47]. In der überarbeiteten Gefahrstoffverordnung (GefStoffV vom 26. November 2010 [BGBl. I S. 1643], geändert durch Artikel 2 des Gesetzes vom 28. Juli 2011 [BGBl. I S. 1622]) sind die Einstufung und Kennzeichnung von Gefahrstoffen vorgeschrieben sowie die Schutzmaßnahmen am Arbeitsplatz festgelegt. Grenzwerte für diese Stoffe spiegeln das gesundheitliche Risiko für den Arbeitnehmer wider.

In Deutschland werden die Grenzen für bestimmte Schadstoffe von der MAK-Kommission der Deutschen Forschungsgemeinschaft festgelegt. Die Grenzwerte für Narkosegase sind in - Tab. 2, vergleichend zu den internationalen Grenzwerten, aufgelistet.

Ziel der arbeitshygienischen Messungen und messtechnischen Überwachung an Anästhesiearbeitsplätzen ist die Einhaltung von Grenzwerten nach TRGS (Technische Regeln für Gefahrstoffe) 900, TRGS 402 und die Einhaltung von Bewertungsindizes für Schadstoffgemische nach TRGS 403. Richtlinien und Anforderungen zur Untersuchung von Gefahrstoffen in geschlossenen Räumen sind in den technischen Regeln für Gefahrstoffe (TRGS 440) aufgelistet. Nach TRGS 403 erfolgt eine Arbeitsplatzanalyse. Die Bewertung von Arbeitsplätzen mit einer Gefahrstoffexposition unterliegt der GefStoffV (§16 „Ermittlungspflicht“). Nach TRGS 402 ist der Arbeitgeber verpflichtet, regelmäßige Kontrollmessungen in Abhängigkeit von der Höhe des Expositionsindexes durchzuführen. Messungen der Narkosegaskonzentrationen bei Vorliegen eines Expositionsindexes > 0,1 (Schwellenwert der MAK; TRGS 402, TRGS 403) sollten in regelmäßigen Zeitabständen veranlasst werden. 
Tab. 2 Internationale Grenzwerte in ppm (parts per million) für volatile Anästhetika.

\begin{tabular}{|c|c|c|c|c|c|c|}
\hline Land & Halothane & Enflurane & Isoflurane & Sevoflurane & Lachgas & Desflurane \\
\hline Dänemark & & - & - & - & - & - \\
\hline Frankreich & 50 & 75 & - & - & - & - \\
\hline Deutschland & 5 & 20 & 10 & - & 100 & - \\
\hline Italien & 2,5 & 5 & - & - & - & -1 \\
\hline Norwegen & 5 & 2 & 2 & 2 & 100 & - \\
\hline Schweden & 5 & 10 & 10 & - & - & \\
\hline Schweiz & 5 & 10 & 10 & - & 100 & - \\
\hline USA NIOSH & 2 & 2 & 2 & 2 & - & - \\
\hline Österreich & 5 & - & - & - & - & - \\
\hline Belgien & 50 & 75 & - & - & 50 & - \\
\hline Finnland & 1 & - & - & - & - & - \\
\hline
\end{tabular}

${ }^{1}$ Aufstellung eines MAK-Wertes wird diskutiert (DFG 2011). Quelle: http://www.dfg.de/download/pdf/dfg_im_profil/gremien/senat/arbeitsstoffe/barbl11.pdf

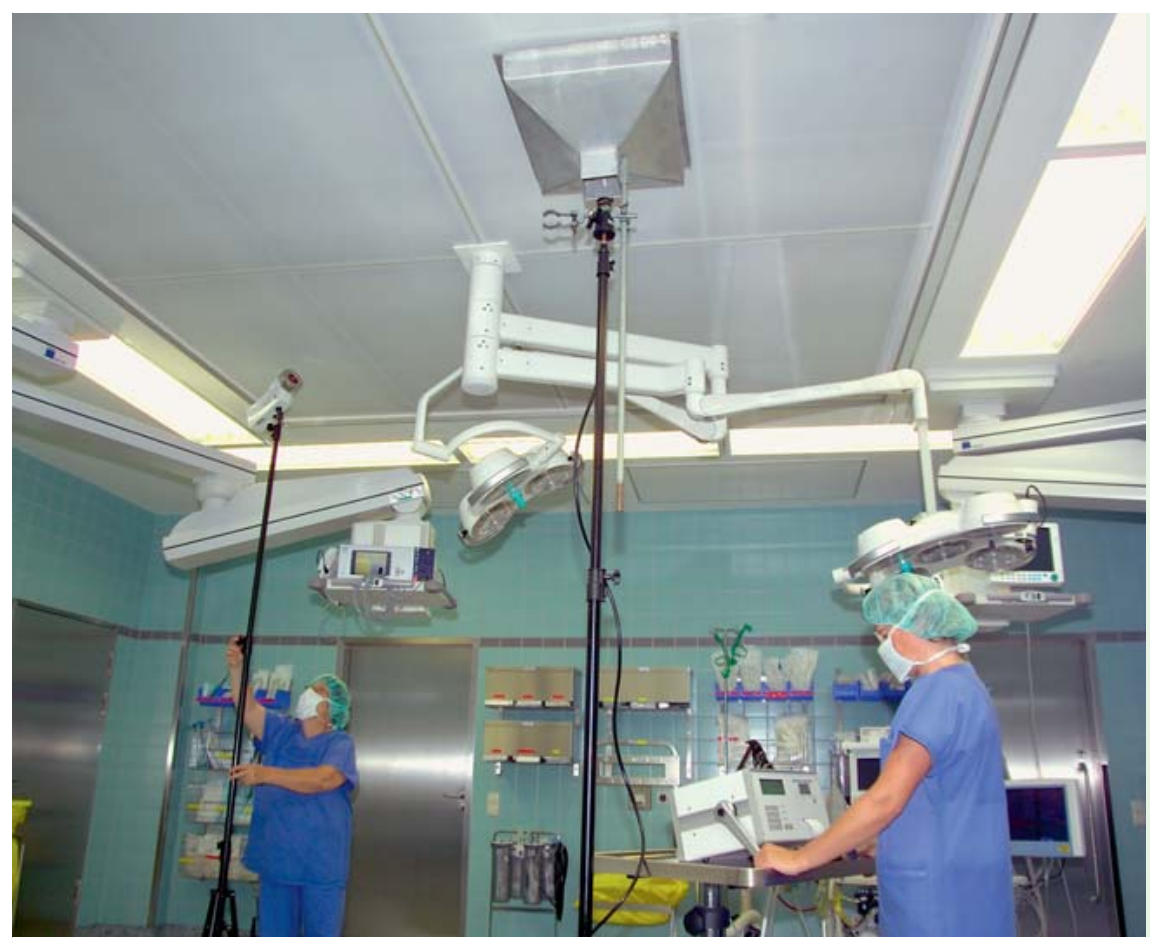

Abb. 4 Arbeitshygienische Messungen im OP-Bereich.

Bei der Gefährdungsbeurteilung des Arbeitsplatzes OP-Raum wird eine messtechnische Überwachung der Räume durchgeführt ( Abb.4); aber vor allem gerade dann, wenn das OPPersonal gesundheitliche Beeinträchtigungen äußert.

Die Raumluftmessungen können personen- oder arbeitsplatzbezogen vorgenommen werden. Mithilfe des Gasmonitors und der Gaschromatografie kann man gleichzeitig mehrere Gase in der Raumluft (Multigasmessung) analysieren. Zu den Methoden der Raumluftmessungen gehören:

- direktanzeigende Verfahren (bei Lachgas, Isofluran und Enfluran),

- Sammelverfahren (PAS-System) auf Aktivkohle-Phasen mit Gaschromatografie (bei Lachgas, Halothan, Isofluran, Enfluran, Desfluran, Sevofluran) bzw. Infrarot-Analyse (IR) und

- Diffusionssammler auf Aktivkohle-Basis mit anschließender Desorption und IR-Analyse (bei Lachgas) [10].

Das biologische Monitoring ist eine weitere Möglichkeit, die zur quantitativen Bestimmung der Belastung durch Schadstoffe eingesetzt werden kann. Bei Einhaltung der arbeitshygienischen
Maßnahmen ist es jedoch nicht notwendig. Beim Vorliegen von gesundheitlichen Beschwerden soll der Betriebsarzt diese Messung in Erwägung ziehen.

Ein biologisches Monitoring beim OP-Personal nach erfolgter Exposition gegenüber volatilen Anästhetika ist nur für Halothan sinnvoll. Hier gibt es nach TRGS 903 einen BGW: 2,5 mg/l Trifluoressigsäure im Vollblut. AGW liegt bei 5 ppm Halothan in der Luft am Arbeitsplatz. Aus den anderen volatilen Anästhetika - Enfluran, Isofluran, Sevofluran, Desfluran - bilden sich nur geringfügig Metaboliten. Deshalb ist die Rückschlussmethodik der MAK-Bestimmung (jetzt BGW) nicht möglich [45]. Eine Übersicht über experimentelle Untersuchungen zum Anästhesiegasmonitoring im Blut und Urin des exponierten Personals findet man bei Rüegger et al. [9]. Von personenbezogenen Messungen wird nur vereinzelt berichtet [8]. Für Lachgas gibt es Hinweise, dass eine Korrelation zwischen der Raumluftbelastung mit $\mathrm{N}_{2} \mathrm{O}$ und der Urinbelastung besteht [10]. 


\section{Arbeitsschutzmaßnahmen bei Expositionen} durch volatile Anästhetika

Die Reihenfolge der Arbeitsschutzmaßnahmen orientiert sich an der Rangfolge der Schutzmaßnahmen gemäß § 19 GefStoffV.

$\mathrm{Zu}$ einer Arbeitsplatzbewertung gehören eine aktuelle Literaturrecherche, zuverlässige Expositionsberechnungen, Raumluftmessungen sowie biologisches Monitoring [10].

Neben der Einhaltung der Grenzwerte für Anästhesiegase und Reduktion der Emissionsbelastung auf das technisch mögliche Minimum [48] am OP-Arbeitsplatz sowie Substitution von Gefahrstoffen (z.B. Ersetzung gefährlicher Stoffe wie Halothan durch Sevofluran oder Isofluran) sollen für die komplette Durchführung der Maßnahmen der Primärprävention weitere STOPMaßnahmen zum Schutz des OP-Personals getroffen werden.

$\mathrm{Zu}$ diesen STOP-Maßnahmen der Verhältnis- und Verhaltensprävention gehören $[8,9,24]$ :

\section{Technische Maßnahmen}

- lüftungstechnische oder technisch-raumorientierte Maßnahmen:

- Raumventilation im OP-Saal (in Neubauten: Optimal wäre pro Stunde die Zuführung von mindestens $1200 \mathrm{~m}^{3}$ Außenluft [DIN 1946 Teil 4 Punkt 5.6], d.h. aufbereitete Frischluft ohne Umluftzusatz; in bereits bestehenden Räumen: mindestens ein 5- bis 6-facher Luftwechsel [Frischgas/Stunde]; es ist vorgeschrieben, eine einmalige jährliche Kontrolle der Lüftungsanlage im OP-Saal [Zu- und Abluftmessungen] durchzuführen)

- Bei Neubauten ist die Installation von RLT-(raumlufttechnischen-)Anlagen [49] in OP-, Ein- und Ausleiträumen sowie in Aufwachräumen erforderlich.

- Ermittlung des technischen Raumstatus (Pflicht im OP-Bereich); hier wird regelmäßig, d.h. einmal jährlich außerhalb des OP-Programms die Grundbelastung des Raumes durch Lachgas bestimmt.

- gerätetechnische Maßnahmen, damit die Außenluftbelastung durch Anästhesiegase reduziert wird:

- Dichtigkeitsprüfungen zur Verminderung von Leckagen (regelmäßige Wartung durch sachkundiges Fachpersonal wird empfohlen); das Ergebnis der Prüfung auf Dichtigkeit des Hochdruckleitungssystems ist schriftlich festzuhalten (DIN 13260 und $\S 22$ der Unfallverhütungsvorschrift [UVV] „Gase“, Berufsgenossenschaftliche Vorschrift [BGV] B 6 [ehemalige VBG 61])

- aktive Anästhesiegasabsaugung (regelmäßige Prüfung der Absaugleistung und Dichtigkeit der Verbindungsstücke und Schläuche des Anästhesiegeräts)

• Niedrigflussanästhesie („Low-Flow“-Anästhesie) und Minimal-Flow-Anästhesie; es ist zu empfehlen, Geräte ohne entsprechende Einrichtung nicht mehr zu bestellen

- Lokalabsaugung nahe an einer Gasaustrittsstelle und Verwendung der Doppelmaske

- Nutzung von Intubationsnarkosen

- Verzicht auf die Verwendung von Anästhesiegasfiltern (Verwendung nur im Sinne einer Notlösung)

- Optimierung der Anästhesietechnik

- Wartung der Geräte (halbjährliche sicherheitstechnische Kontrolle der Geräte nach Medizingeräteverordnung [MedGV])

- messtechnische Überwachung (Feststellung durch Arbeitsbereichsanalysen nach TRGS 402 und TRGS 403) zur Einhal- tung der Grenzwerte bzw. des Bewertungsindexes für Gemische:

- Durchführung der Arbeitsbereichsanalyse (Erfassung der Gefahrstoffe, Expositionsmessungen, Beschaffung des Grundwissens)

- Dauerüberwachung mit einem kontinuierlich messenden $\mathrm{N}_{2} \mathrm{O}$-Messgerät (die Messergebnisse sind 30 Jahre aufzubewahren).

\section{Organisatorische Maßnahmen}

- Hinweise und Informationen für die Mitarbeiter

- Einweisung und Ausbildung neuer Mitarbeiter

- Durchführung der regelmäßigen Unterweisungen (§20 [2] GefStoffV, TRGS 555) vor Aufnahme der Tätigkeit und danach mindestens einmal jährlich mündlich; Inhalt und Zeitpunkt der Unterweisung sind schriftlich festzuhalten und von den Eingewiesenen durch Unterschrift zu bestätigen. Die Nachweise sind 2 Jahre aufzubewahren.

- Festlegung der Überwachung, Gerätekontrollen und Dichtigkeitsprüfungen.

\section{Persönliche Maßnahmen}

- leckarme Arbeitstechniken und Systeme

- sorgfältige Maskenhandhabung durch entsprechende Arbeitsweise des Anästhesiepersonals

Die Wirksamkeit aller angeführten Schutzmaßnahmen muss entsprechend $\S 18$ GefStoffV, TRGS 402 und TRGS 403 überwacht werden.

\section{Arbeitsmedizinische Vorsorge unter dem Aspekt der Anästhesiegasbelastung}

In Deutschland werden beim OP-Personal arbeitsmedizinische Vorsorgeuntersuchungen nach G42 „Tätigkeit mit Infektionsgefährdung“ durchgeführt. Eine gezielte Vorsorge wegen Anästhesiegasexposition ist nicht vorgeschrieben. Es existiert kein Grundsatz für die arbeitsmedizinische Vorsorge beim Umgang mit Anästhesiegasen. Deshalb ist zu empfehlen, dass sich jeder/ jede Mitarbeiter/-in des OP- und Anästhesiebereichs durch den Personalarzt unter dem Aspekt der Anästhesiegasbelastung untersuchen lässt.

Anästhesiegasexposition und Schwangerschaft ist ein Thema, mit dem die Arbeitsmediziner ständig konfrontiert werden. Es ist zu empfehlen, dass schwangere OP-Mitarbeiterinnen so schnell wie möglich einen Betriebsarzt aufsuchen und sich zu Fragen von Schwangerschaft und Exposition durch Anästhesiegase informieren lassen. Die betroffenen Mitarbeiterinnen sollten nur in OPSälen arbeiten, in denen

1. die Grenzwerte bei Narkoseverfahren unterschritten sind und 2. kein Halothan verwendet wird.

Für die Durchsetzung des Mutterschutzes sind in jedem Bundesland Gewerbeaufsichtsämter oder Ämter für Arbeitsschutz zuständig. Leider werden die Gefährdungen durch volatile Anästhetika in verschiedenen Bundesländern nicht homogen beurteilt [10].

\section{Chirurgische Rauchgase}

$\nabla$

Chirurgische Rauchgase oder Gasgemische entstehen bei Anwendung verschiedener chirurgischer Verfahren, bei denen z.B. „gelasert" oder "gekautert" wird im Sinne der Thermokoagulation, beim Schneiden, zum Verschließen von Gefäßen, bei der Vapori- 
sation oder bei Fotoablation [11]. Sie stellen eine komplexe Mischung von biologischen und zellulären Inhaltsstoffen, gas- und dampfförmigen sowie partikulären Schadstoffen dar. Bei der thermischen Zersetzung der Gewebe können außerdem neue Abbrandprodukte in Form von Rauch und Dämpfen entstehen. Die Substanzen können sich auf der Partikeloberfläche einlagern. Oft können diese auch in den Tröpfchen gelöst sein. Das alles vermischt sich mit der Luft zu einem Aerosol (engl.: Laser Generated Airbone Contaminants). Die Zusammensetzung des Rauches ist je nach eingesetztem Verfahren und behandeltem Gewebe unterschiedlich [50].

\section{Chirurgische Rauchgase und deren Wirkung}

Von der Arbeitsgruppe der Sektion Gesundheitswesen der Internationalen Vereinigung für Soziale Sicherheit (IVSS) wurde eine Gefährdungsbeurteilung der Tätigkeiten mit Exposition gegenüber chirurgischen Rauchgasen vorgenommen [51,52]. Die Datenlage zur potenziellen Gefährdung durch chirurgische Rauchgase ist miserabel. Die Partikel in dieser Mischung sind von unterschiedlicher Größe: von < $10 \mathrm{~nm}$ bis zu mehr als $200 \mu \mathrm{m}$; das hängt vom chirurgischen Verfahren und von der Intensität der Energieeinwirkung ab. Ganz kleine Partikeldurchmesser $<0,1 \mu \mathrm{m}$ entstehen bei Einsatz von Elektrokautern, bei Laserbehandlung zur Gewebeabtragung beträgt der Durchmesser der Rauchpartikel ca. $0,3 \mu \mathrm{m}$, beim Ultraschallskalpell ist der mittlere Partikeldurchmesser 0,35 bis 6,5 $\mu \mathrm{m}$. Die Partikel dieser Größenordnung können eingeatmet werden und sind alveolengängig. Mit 95\% gehört der Wasserdampf zum Hauptbestandteil. Die chemische Analyse der Mischung zeigt, dass organische Pyrolyseprodukte (aromatische Kohlenwasserstoffe, Cyanwasserstoffe, Formaldehyd und polyzyklische aromatische Kohlenwasserstoffe) vorhanden sein können. Außerdem entstehen bei elektrochirurgischen Verfahren anorganische Schadstoffe (z.B. Kohlenstoffoxide wie $\mathrm{CO}$ und $\mathrm{CO}_{2}$, Schwefel- und Stickoxide, Ammoniak u. a.).

Bei den biologischen Inhaltsstoffen können u.a. Viren (z. B. Humanes Papilloma-Virus [HPV]) bzw. virale DNS-Fragmente, lebensfähige Bakterien, aber auch lebensfähige Zellen vorkommen. Damit birgt die Exposition durch chirurgische Rauchgase potenzielle Gefährdungen toxischer und infektiöser Natur. Die beim Einsatz des Elektrokauters entstehenden Abbrandprodukte entsprechen in ihrem Gefährdungspotenzial dem Laser-Aerosol. Beim Kautern entsteht fast doppelt so viel Rauch pro Gewebemenge wie beim Lasern [53].

Einige der chemischen Komponenten besitzen eine schleimhautreizende, zell- und gentoxische sowie mutagene oder allergene Potenz [53]. Die Frage, ob ihre Dosis im Laser-Aerosol ausreicht, um diese Wirkungen bei Exponierten wirklich zu erzeugen, ist nicht geklärt und bedarf einer weiteren Forschung.

Unter Lasereinwirkung sollen Fluorchlorkohlenwasserstoff (FCKW)-haltige Anästhesiegase ihre Halogensubstituenten abspalten, was ein zusätzliches Gefährdungspotenzial darstellen kann. Diese Radikale können mit organischem Material der Gewebe reagieren, dabei entstehen die extrem schleimhautreizenden Halogenwasserstoffsäuren [53].

Die Bestandteile der Rauchgase können dosisabhängig neurologische Beschwerden hervorrufen wie z. B. Kopfschmerzen, Schwächegefühl, Übelkeit usw. Auch starke Geruchsbelästigung und Reizreaktionen der Atemwege oder der Augen können vorkommen. Ein erhöhtes Krebsrisiko bei chronischen Wirkungen ist nicht auszuschließen.
Calero et Brusis [54] berichten über die erstmalige Anerkennung der Larynxpapillomatose als Berufskrankheit bei einer OPSchwester.

In der Literatur sind nur wenige deutsche Quellen zum Thema Laser-Aerosol zu finden [55,56]. Die Daten der STILMED-Studie [57] bilden die Basis für die arbeitsmedizinische Bewertung der Gefährdungspotenziale für das Personal im OP-Bereich.

\section{Arbeitshygienische Beurteilung der Exposition durch chirurgische Rauchgase}

Laser-Aerosol aus Humangewebe ist ein Bioaerosol i.S. der Technischen Regeln für Biologische Arbeitsstoffe TRBA 500 (TRBA 500 vom 01.05.1999, BArbBl Nr.6/99, Seite 81). Die sind nach der Biostoffverordnung und nach der EU-Biostoff-Richtlinie 2000/ 54/EG der Risikogruppe 2 zuzuordnen.

\section{Arbeitsschutzmaßnahmen bei Expositionen \\ durch chirurgische Rauchgase}

Auch bei dieser Gefährdung sind Arbeitsschutzmaßnahmen angesagt, deren Reihenfolge, wie schon oben beschrieben wurde, nach der

Substitution mit den

Technischen Arbeitsschutzmaßnahmen beginnt (Vermeidung einer Gefährdung, Einsatz technischer Schutzmaßnahmen wie z.B. Quellen- bzw. Lokalabsaugungen chirurgischer Rauchgase [möglichst dicht an der Quelle], chirurgische Absaugsysteme mit Einmalfilter, mobile Rauchgasabsaugungen, angepasstes Handstück mit integrierter Absaugdüse; technische Raumlüftungen [RLT-Anlage nach DIN 1946 Teil 4]), dann

Organisatorische Schutzmaßnahmen (z. B. Einsatzpläne, Überwachung der Wirksamkeit von Schutzmaßnahmen, Information für die Beschäftigten, regelmäßige Unterweisung der Handelnden), und

Persönliche Schutzmaßnahmen (bei chirurgischen Eingriffen z. B. Atemschutzmaske wie partikelfiltrierende Halbmasken gemäß EN 149: 2001 + A1: 2009 [mindestens Filterklasse FFP 2] [58], bei Wartungsarbeiten z.B. Filterwechsel mit üblichen hygienischen Schutzmaßnahmen).

Auch in diesem Fall soll die Überwachung der Wirksamkeit von Schutzmaßnahmen bei Belastungen durch chirurgische Rauchgase entsprechend $\S 18$ der GefStoffV erfolgen.

Die Exposition des OP-Personals durch chirurgische Rauchgase ist von dem verwendeten OP-Gerät, der Rauchgasabsaugung, der RLT-Anlage, von der Wechselrate der Allgemeinbelüftung, den organisatorischen Maßnahmen sowie den Fähigkeiten und Fertigkeiten des Operateurs abhängig. Die Prüfung der quantitativen Zusammensetzungen der chirurgischen Rauchgase sollte in regelmäßigen Zeitabständen veranlasst werden. Individuelle Expositionsmessungen sind jedoch nicht hilfreich [12].

\section{Arbeitsmedizinische Vorsorge unter dem Aspekt der Belastung durch chirurgische Gase}

Eine gezielte spezifische arbeitsmedizinische Vorsorgeuntersuchung bei OP-Personal mit den Arbeitsbelastungen chirurgischer Rauchgase ist z.Z. nicht vorgesehen und wird routinemäßig nicht durchgeführt. Es sind auch keine Vorsorgeprogramme bekannt, wobei bei der allgemeinen Vorsorgeuntersuchung bzw. bei der arbeitsmedizinischen Vorsorgeuntersuchung nach G42 „Tätigkeit mit Infektionsgefährdung“ eventuelle Vorerkrankungen erfasst werden sollten. 
Fazit

$\nabla$

Eine weitere enge Zusammenarbeit der Betriebsmediziner und des leitenden OP-Personals ist wünschenswert. Der „fachkundige Arzt“ bzw. ein „beauftragter Arzt für Arbeitsschutz am anästhesiologischen Arbeitsplatz" soll von dem Betriebsarzt und der Sicherheitskraft informiert werden, ob die Luftgrenzwerte eingehalten und die Vorgaben der Technischen Regeln für Gefahrstoffe umgesetzt sind, sowie über die Überwachung von Arbeitsbereichen beraten werden. Er soll über allgemeine Regeln des Arbeitsschutzes, arbeitsschutzrelevante Kenntnisse von Narkosetechnik, Narkosegeräten und Lüftungstechnik verfügen. Außerdem soll er über die Durchführung und Befunderhebung der Arbeitsbereichsanalyse informiert sein. Trotz des vielleicht initial nicht unbedingt erkennbaren Risikos sollen die arbeitshygienischen Grenzwerte eingehalten werden, damit weder eine Gefährdung für das OP-Personal besteht noch gesundheitliche (auch wenn nur evtl. subjektive) Beeinträchtigungen eintreten.

Schlussfolgernd hat die interdisziplinäre Zusammenarbeit der Arbeits- und Betriebsmedizin sowie der operativen (und insbesondere auch der chirurgischen) Fächer bzw. der Anästhesie eine große Bedeutung. Eine wichtige gemeinsame Aufgabe der Führungskräfte und Hochschullehrer in der Chirurgie, Anästhesie und Arbeitsmedizin für die Zukunft ist es, schon bei der Ausbildung des chirurgischen OP- und Anästhesiepersonals die Fragen des Arbeitsschutzes zu implementieren, um die potenziellen Belastungen durch Anästhesie- und chirurgische Rauchgase für die Beschäftigten zu verdeutlichen. Auch das Personal ist auf angezeigte präventive Verhaltensweisen aufmerksam zu machen.

Die Anästhesie- und chirurgischen Rauchgase können potenziell aufgrund der toxischen Eigenschaften gesundheitsschädliche Auswirkungen auf das medizinische Personal haben, wenn die Schutzmaßnahmen am Arbeitsplatz ungenügend sind. In Deutschland werden die Arbeitsschutzmaßnahmen streng eingehalten. Deswegen, aber auch weil eine anhaltende arbeitsmedizinische Sensibilisierung in Zusammenarbeit mit den o.a. relevanten Fächern auf die vorgetragene Thematik erfolgt sowie in den letzten 10-15 Jahren neue Entwicklungen im Rahmen technisierter operativer Eingriffe inauguriert wurden, sind die potenzielle Belastungen durch Anästhesie- und chirurgische Rauchgase in Deutschland eher reduziert worden.

Interessenkonflikt: Nein

\section{Literatur}

1 Hofmann F. Betriebsarzt im Krankenhaus. Infektionsprophylaxe, Begehungen und Ergonomie. 3. Aufl. Landsberg/Lech: ecomed; 2000

2 Matern $U$, Koneczny S, Scherrer $M$ et al. Arbeitsbedingungen und Sicherheit am Arbeitsplatz OP. Dtsch Ärztebl 2006; 103: A3187-A3192

3 Bauer H. Arbeitsplatz OP: Realität und Anspruch. Dtsch Ärztebl 2006; 103: A3185-A3186

4 Effert J. Aktuelle Gesichtspunkte zur Narkosegasbelastung am Arbeitsplatz. Die Schwester - Der Pfleger 1995; 6: 252-529

5 Hoerauf K, Mayer T, Hobbhahn J. Arbeitsplatzbelastung durch Enfluran und Lachgas in Operationsräumen. Zentralbl Hyg Umweltmed 1996; 3 : 265-274

6 Dudziak R. Nebenwirkungen von flüchtigen Anästhetika auf das Anästhesiepersonal unter besonderer Berücksichtigung des Mutterschutzgesetzes. Anästhesiol Intensivmed 1981; 22: 81-92

7 Conzen P. Gesundheitliche Risiken von Inhalationsanästhetika. AINS 1994; 29: 10-17

8 Westphal K, Martens S, Lischke V et al. Arbeitsplatzbelastung mit Inhalationsanästhetika in der Herzchirurgie. Z Herz Thorax Gefaesschir 1997; $11: 244-248$
9 Rüegger M, Jost M, Meier A et al. Umgang mit Anästhesiegasen. Gefährdung, Schutzmaßnahmen. Luzern: SuVa; 2000

10 IVSS, Sektion Gesundheitswesen. Umgang mit Anästhesiegasen im Gesundheitswesen. Beitrag Deutschland. U. Eickmann. BGW, Stand 03/ 2001

11 Merz B, Rüegger M, Käslin E et al. Factsheet: Chirurgische Rauchgase Gefährdungen und Schutzmaßnahmen. Suva. Version März 2011. Im Internet: www.suva.ch/arbeitsmedizin-factsheets; Stand: 02.02.2012

12 Eickmann U. Chirurgische Rauchgase: Gefährdungen und Schutzmaßnahmen. Vortrag. 24. Freiburger Symposium, 15.-17. September 2010 in Freiburg. Im Internet: http://www.bgw-online.de/internet/generator/Inhalt/OnlineInhalt/Medientypen/Fachartikel/chirurgische_Rauchgase.html; Stand: 05.03.2012

13 Kelling G. Über die Beseitigung der Narkosedämpfe aus dem Operationssaale. Zentralbl Chir 1918; 45: 602-606

14 Hirsch JB, Kappurs AL. Über die Mengen des Narkoseäthers in der Luft von Operationssälen. Z Hyg 1929; 110: 391-398

15 Schwenzer N, Ehrenfeld M. Allgemeine Chirurgie. Bd. 1, Zahn-, Mund-, Kiefer-Heilkunde. Lehrbuch zur Aus- und Weiterbildung. 3. Akt. und erweiterte Auflage. Stuttgart, New York: Georg Thieme Verlag; 2000

16 Loscar M, Conzen P. Volatile Anästhetika. Der Anaesthesist 2004; 53: 183-197

17 Winter CG, Lamprecht E, Hamm G et al. Arbeitstoxikologische Studie zur Einschätzung des expositionellen Risikos des Operationspersonals gegenüber Inhalationsanaesthetika. Z Ges Hyg 1987; 33: 622-626

18 Hagemann $H$. Arbeitsplatzbelastungen durch volatile Anästhetika. In: Bundesarbeitsgemeinschaft für Arbeitssicherheit (BASI) (Hrsg.). Auf einen Blick A + A89 1990, Kongressband 156

19 Hagemann $H$, Winter CG. Arbeitsmedizinische Überlegungen zum Anästhesie-Arbeitsplatz. Beitr. Anaesth Intens Notfallmed 1993; 42: 41-53

20 Ganiere-Monteil C, Pineau A, Souron $R$ et al. Halothane et enflurane dans les blocs opératoires: exposition et prévention. Arch Mal Prof 1993; 54: 563-568

21 Candelaria LM, Smith RK. Propofol Infusion Technique for Outpatient General Anesthesia. J Oral Maxillofac Surg 1995; 53: 124-128

22 Lucchini R, Placidi D, Toffoletto F et al. Neurotoxicity in operating room personnel working with gaseous and nongaseous anesthesia. Int Arch Occup Environ Health 1996; 68: 188-192

23 Marx T. Belastung des Arbeitsplatzes und der Umwelt mit Narkosegasen. Arbeitsmed Sozialmed Umweltmed 1998; 33: 64-75

24 Müller-Bagehl S (Verfass.) Umgang mit Narkosegasen. u. Mitarb. In: M. Diefenbach, H. Reinhold, U. Schröter B. Wüstefeld. Merkblatt M9, 3. Aufl. Hrsg.: Freie und Hansestadt Hamburg: Behörde für Wissenschaft und Gesundheit, Amt für Arbeitsschutz; 1997

25 Bittighofer PM. Leserfrage. Einsatz von schwangeren Mitarbeiterinnen im OP-Bereich unter besonderer Berücksichtigung der Exposition gegenüber Narkosegasen. Antwort 1. Arbeitsmed Sozialmed Umweltmed 2004; 39: 496-497

26 Eickmann U, Remé T. Leserfrage. Einsatz von schwangeren Mitarbeiterinnen im OP-Bereich unter besonderer Berücksichtigung der Exposition gegenüber Narkosegasen. Antwort 2. Arbeitsmed Sozialmed Umweltmed 2004; 39: 497-498

27 Bonnekoh B, Stötzel B, Böckelmann R et al. Allergologische Aspekte am anästhesiologischen Arbeitsplatz. Anaesth Int Med 2004; 9: 516-521

28 Greim H, Reutter U. Leserfrage. Einsatz von schwangeren Mitarbeiterinnen im OP-Bereich unter besonderer Berücksichtigung der Exposition gegenüber Narkosegasen. Antwort 3. Arbeitsmed Sozialmed Umweltmed 2004; 39: 498-499

29 Zühlsdorff A. Vergleich der Arbeitsplatzbelastung während einer Xenon- oder Lachgasanästhesie. Diss., Med. Fakultät der Rheinisch-Westfälischen Technischen Hochschule Aachen; 2009

30 Gutmann L, Johnsen D. Nitrous oxide-induced myeloneuropathy: report of cases. JADA 1981; 103: 239-241

31 Brodsky JB, Cohen E, Brown BW et al. Exposure to nitrous oxide and neurologic disease among dental professionals. Anesth Analg 1981; 60: 297-301

32 Saurel-Cubizolles MJ, Estryn-Behar M, Maillard MF et al. Neurophysiological symptoms and occupational exposure to anaesthetics. $\mathrm{Br}$ J Industr Med 1992; 49: 276-281

33 Bruce DL, Bach MJ. Effects of trace anaesthetic gases on behavioural performance of volunteers. Br J Anaesth 1976; 48: 871-876

34 Smith G, Shirley AW. Failure to demonstrate effect of trace concentrations of nitrous oxide and halothane on psychomotor performance. $\mathrm{Br}$ J Anaesth 1977; 49: 65-70 
35 Venables H, Cherry N, Waldron HA et al. Effects of trace levels of nitrous oxide on psychomotor performance. Scand J Work Environ Health 1983; 9: 391-396

36 Stollery BT, Broadbent DE, Lee WR et al. Mood and cognitive functions in anaesthetic working in actively scavenged operating theatres. $\mathrm{Br}$ J Anaesth 1988; 61: 446-455

37 Cook TL, Smith M, Starkweather JA et al. Behavioral effects of trace and subanesthetic halothane and nitrous oxide in man. Anesthesiology 1978; 49: 419-424

38 Peric $M$, Vranes $Z$, Marusic $M$. Immunological disturbances in anaesthetic personnel chronically exposed to high occupational concentrations of nitrous oxide and halothane. Anaesthesia 1991; 46: 531-537

39 Ziv Y, Shohat B, Baniel J et al. The immunologic profile of anesthetists. Anesth Analg 1988; 67: 849-851

40 Cohen EN, Brown BW. Occupational disease in dentistry and chronic exposure to trace anaesthetic gases. JADA 1980; 101: 21-31

41 Lings $S$. Halothane related liver affection in an anaesthetist. Br J Industr Med 1988; 45: 716-717

42 Rowland AS, Baird DD, Weinberg $C R$ et al. Reduced fertility among women employed as dental assistants exposed to high levels of nitrous oxide. N Engl J Med 1992; 327: 993-997

43 Schneider $H$. Psychodiagnostische Untersuchungsergebnisse nach chronischer Halothanexposition - Ein Erfahrungsbericht. Z Ges Hyg 1986; 32: 104-106

44 Wegner R, Rinker G, Poschadel B et al. Zur Halothanbelastung von Operationspersonal in Abhängigkeit von raumlufttechnischen Bedingungen. Arbeitsmed Sozialmed Präventivmed 1990; 25: 264-270

45 Hagemann $H$, Winter CG. Narkosegasbelastungen am Arbeitsplatz Anästhesie - Differenzierungsmöglichkeiten und Belastungsstrukturen. Anästh Intensivmed 1994; 35: 191-197

46 Schwettmann RS, Casterline CL. Delayed asthmatic response following occupational exposure to enflurane. Anesthesiology 1976; 44: 166169

47 Grenzwertliste 2011. Sicherheit und Gesundheitsschutz am Arbeitsplatz. IFA-Report 1/2011. DGUV Deutsche Gesetzliche Unfallversicherung, Spitzenverband. Berlin: DCM - Druckcenter Meckenheim; 2011
48 Pothmann W, Shimada K, Goerig M et al. Belastungen des Arbeitsplatzes durch Narkosegase. Anaesthesist 1991; 40: 339-346

49 Kramer A, Külpmann $R$, Wille $F$ et al. Infektiologische Bedeutung von Raumlufttechnischen Anlagen (RLTA) in Operations- und Eingriffsräumen. Zentralbl Chir 2010; 135: 11-17

$50 \mathrm{Al}$ Sahaf OS, Vega-Carrascal I, Cunningham FO et al. Chemical composition of smoke produced by high-frequency electrosurgery. Ir J Med Sci 2007; 176: 229-232

51 IVSS, Internationale Sektion der IVSS für die Verhütung von Arbeitsunfällen und Berufskrankheiten im Gesundheitswesen: Eickmann U, Felcy M, Fokohl I, Rüegger M, Bloch M. Chirurgische Rauchgase - Gefährdungen und Schutzmaßnahmen, Hamburg 2010; Im Internet: www.suva.ch; Stand: 02.02.2012

52 Eickmann U, Falcy M, Fokuhl I et al. Chirurgische Rauchgase - Gefährdungen und Schutzmaßnahmen. Arbeitsmed Sozialmed Umweltmed 2011; 46: 14-23

53 Abbrandprodukte in der Laser- und HF-Chirurgie. Eine Informationsbroschüre für medizinisches Personal und Patienten. Atmos, Medizin Technik. Im Internet: www.atmosmed.com/html/seiten/Rauchabsaugungsfibel_AtmoSafe.pdf; Stand: 05.10.2012

54 Calero L, Brusis T. Larynxpapillomatose - erstmalige Anerkennung als Berufskrankheit bei einer OP-Schwester. Laryngo-Rhino-Oto 2003; 82: 790-793

55 Bindig $U$, Wäsche $U$. Analyse von Abbrandprodukten beim Einsatz medizinischer Laser. In: Berlien H-P, Müller GJ (Hrsg.) Angewandte Lasermedizin. 13. Erg. Lfg. Landsberg: Ecomed; 1997

56 Wäsche W. Sicherheits- und Schutzmaßnahmen zur Reduzierung bzw. Abwendung von potentiellen Gefährdungen durch Laser-Pyrolyseprodukte. Berlien HP, Müller G, Hrsg. Angewandte Lasermedizin. 13. Erg. Lfg. Landsberg: Ecomed; 1997

57 STILMED-Studie (Safety Technology in Laser Medicine), EUREKA-Verbundprojekt EU-642, 1991-1995. „Bewertung von Abbrandprodukten bei der medizinischen Laseranwendung“. Hrsg. Berlin: VDI-Technologiezentrum für Physikalische Technologien; 1997

58 EN 129. Atemschutzgeräte - Filtrierende Halbmasken zum Schutz gegen Partikel - Anforderungen, Prüfung, Kennzeichnung; Deutsche Fassung EN 149: $2001+$ A 1: 2009 